\title{
Tocilizumab therapy in rheumatoid arthritis with interstitial lung disease: a multicenter retrospective study
}

Manfredi Andreina $^{a}$, MD, Cassone Giulia ${ }^{a, b, c}$, MD, Furini Federica ${ }^{d}$, MD, Gremese Elisa ${ }^{e}$, MD, Venerito Vincenzo ${ }^{f}, \mathrm{MD}_{\text {, Atzeni Fabiola }}{ }, \mathrm{MD}$, Arrigoni Eugenio ${ }^{h}$, MD, Della Casa Giovanni ${ }^{i}$, MD, Cerri Stefania ${ }^{l}, \mathrm{MD}$, Govoni Marcello ${ }^{d}$, MD, Petricca Luca $^{e}$, MD, Iannone Florenzo ${ }^{f}$, MD, Salvarani Carlo $^{a, c}$, MD, Sebastiani $\mathrm{Marco}^{a}$, MD.

${ }^{a}$ Chair and Rheumatology Unit, University of Modena and Reggio Emilia, Azienda OspedalieroUniversitaria Policlinico di Modena, Modena, Italy.

${ }^{b}$ Clinical and Experimental Medicine PhD Program, University of Modena and Reggio Emilia, Modena, Italy.

${ }^{c}$ Rheumatology Unit, IRCCS Arcispedale Santa Maria Nuova, Azienda Unità Sanitaria LocaleIRCCS di Reggio Emilia, Reggio Emilia, Italy.

${ }^{d}$ Department of Medical Sciences, Division of Rheumatology, Santa Anna University Hospital, Ferrara, Italy.

${ }^{e}$ Division of Rheumatology, Institute of Rheumatology, Catholic University of the Sacred Heart, Rome, Italy.

${ }^{f}$ Rheumatology Unit, University of Bari, Italy.

${ }^{g}$ Rheumatology Unit, University of Messina, Italy.

${ }^{h}$ Rheumatology Unit, ERI Medicine, Hospital of Piacenza, Italy.

${ }^{i}$ Radiology Unit at Azienda Ospedaliera Universitaria Policlinico di Modena, Modena, Italy.

${ }^{l}$ Respiratory Disease Unit, University of Modena and Reggio Emilia, Azienda OspedalieroUniversitaria Policlinico di Modena, Modena, Italy.

\section{Authors' contributions}

All authors have contributed significantly to the article, and they agree with the content of the manuscript.

This article has been accepted for publication and undergone full peer review but has not been through the copyediting, typesetting, pagination and proofreading process which may lead to differences between this version and the Version of Record. Please cite this article as doi: $10.1111 /$ imj.14670 
- Manfredi A. and Sebastiani M. contributed to: study conception and design, manuscript drafting, data analysis or interpretation, critical manuscript revision and final manuscript approval.

- Cassone G. contributed to: data acquisition, manuscript drafting, critical manuscript revision and final manuscript approval.

- Venerito V, Furini F, Iannone F, Gremese E, Atzeni F, Arrigoni E, Govoni M, Petricca L, Salvarani C contributed to: data aquisition, final manuscript revision and approval.

Cerri S, Della Casa G, contributed to: pulmonary data acquisition, final manuscript revision and approval.

Acknowledgements and Conflict of interest: the authors declare they have no conflicts of interest.

Corresponding author:

Dr. Andreina Manfredi, MD

Rheumatology Unit

University of Modena and Reggio Emilia

Via del Pozzo, 71

41100 Modena Italy

Tel +39-059-4225295

Fax +39-059-4223007

E-mail: andreina.manfredi@gmail.com 


\begin{abstract}
Background

Interstitial lung disease (ILD) is the most severe extra-articular manifestation of rheumatoid arthritis (RA). Although it is responsible of $10-20 \%$ of all RA mortality, no controlled studies are available for the treatment of RA-ILD and its therapeutic approach is still debated.
\end{abstract}

\title{
Aims
}

In this study, we analysed the evolution of ILD in a population of RA patients treated with tocilizumab (TCZ).

\section{Methods}

In this national multicenter study, we retrospectively collected patients with RA-ILD treated with at least one dose of TCZ. For each patient, disease activity and serological data were evaluated. Moreover, we analysed the evolution of high-resolution computed tomography (HRCT) and pulmonary function tests, including forced vital capacity (FVC) and diffusing capacity of carbon monoxide (DLCO).

\section{Results}

Twenty-eight RA-ILD patients were identified (females/males 18/10, mean age 61.6 years), with a mean follow-up for TCZ therapy of 30 months. At the end of follow-up, FVC remained stable in 14 patients (56\%), improved in 5 (20\%) and worsened in 6 (24\%). DLCO remained stable in 14 patients (56\%), improved in 5 (20\%) and worsened in 6 (24\%), even though in 3 patients DLCO and FVC showed an opposite trend.

HRCT remained stable in the majority of cases (25), worsened in 2 patients with a usual interstitial pneumonia pattern, improved in only one case with a nonspecific interstitial pneumonia pattern.

\section{Conclusions}

The management of RA-ILD patients remains a critical unmet need. TCZ demonstrated a good safety profile in patients with RA-ILD and a potential effect on the stabilization of the lung involvement.

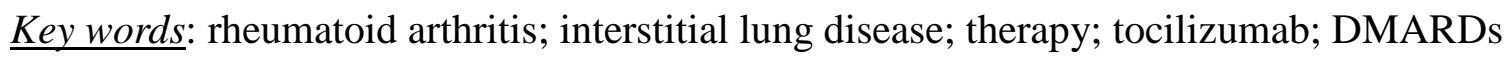

This article is protected by copyright. All rights reserved. 


\section{Introduction}

Rheumatoid arthritis (RA) is a chronic inflammatory disease, characterized by synovial joint swelling and tenderness, with progressive disability and joint destruction ${ }^{1}$.

The interstitial lung disease (ILD) is the most severe extra-articular manifestation of RA, with impact on both therapeutic strategy and overall prognosis and survival ${ }^{2}$.

About $10 \%$ of the RA population develops a clinically significant ILD that is responsible for decreased quality of life and progressive chronic disability, but also of $10-20 \%$ of all mortality associated to the disease, with a mean survival of 5-8 years ${ }^{3-6}$.

Although both genetic and environmental factors have been investigated, the pathogenesis of RAassociated ILD (RA-ILD) remains unclear ${ }^{7-8}$.

Moreover, the majority of conventional and biologic diseases modifying anti-rheumatic drugs (DMARDs) have been associated to the development or progression of ILD ${ }^{9-10}$.

For these reasons, since no controlled studies are available, the therapeutic approach to RA-ILD is still debated and often empirical ${ }^{7,11-13}$. Some limited reports have evaluated the safety and the efficacy of tocilizumab (TCZ), a humanized anti-interleukin 6 (IL6) antibody, in the treatment of patients with RA-ILD ${ }^{14-15}$.

III this retrospective study, we analysed the evolution of ILD in a population of RA patients treated with TCZ.

\section{Patients and methods}

In a national multicenter study, we retrospectively collected patients with ILD associated to RA treated with TCZ. All RA patients attending the Rheumatology Units of 6 Italian centers after 2008 and treated with TCZ for at least 6 months were retrospectively evaluated to identify patients with ILD.

This article is protected by copyright. All rights reserved. 
RA was diagnosed according to the 1987 or 2010 classification criteria depending on the year of diagnosis $^{16,17}$. The study was approved by the local Institutional Review Board.

The different patterns of interstitial lung involvement, defined by pulmonary biopsy or chest high resolution computer tomography (HRCT), were classified according to the standardized criteria of the American Thoracic Society/European Respiratory Society International Multidisciplinary Consensus Classification of the Idiopathic Interstitial Pneumonias ${ }^{18}$ as follows: 1 ) definite or probable usual interstitial pneumonia (UIP), 2) non-specific interstitial pneumonia (NSIP); and 3) organizing pneumonia (OP) and mixed patterns.

RF was determined by nephelometry; anti-cyclic citrullinated peptides antibodies (ACPA) were detected by standard commercial enzyme-linked immunosorbent assays (ELISA).

The results of pulmonary function tests (PFT) were expressed as percentages of the predicted value of each parameter and corrected for age, gender and height. Pulmonary function was considered as abnormal if forced vital capacity (FVC) was $<80 \%$ of predicted values. Single-breath diffusing capacity of the lung for carbon monoxide (DLCO-SB) and DLCO adjusted by the alveolar volume (DLCO-VA) were used to assess gas transfer. The last HRCT and the last PFT performed before starting TCZ were recorded as baseline.

\subsection{Outcome Variables}

A variation of $10 \%$ of FVC or DLCO compared to baseline was considered clinically significant ${ }^{19}$. Improvement, worsening or stability of HRCT was centrally evaluated in a blinded manner by an experienced thoracic radiologist (GDC). PFTs were collected at baseline and periodically assessed and for all patients was recorded the last available value (within 3 months from the end of followup). All patients but one repeated HRCT at the end of follow-up.

\subsection{Statistical Analysis}


Results were expressed as median and interquartile range (IQR). Continuous variables were compared using unpaired or paired nonparametric tests (Mann Whitney or Wilcoxon test, respectively). A p value less than 0.05 was considered significant. Statistical analyses were performed using the SPSS statistical software, version 17.0 (SPSS Inc., Chicago, IL, USA) ${ }^{20}$.

\section{Results}

We included 28 RA-ILD patients (18 females and 10 males, median age 64, IQR 15) treated with TCZ. The drug was administered at the standard dose, both intravenous (8 mg/kg every 4 weeks) and subcutaneously (162 mg weekly). For all patients, HRCT was available in the previous 12 months before the beginning and at the end of the therapy with TCZ, while PFTs were available in 25/28 patients.

Baseline characteristics of RA-ILD patients included in the study are summarized in Table 1.

The median follow-up was 30 months (range 6-90). All patients were positive for RF and all but 2 for ACPA.

An UIP pattern was described in 14/28 patients (50\%), while a NSIP pattern was identified in other 13 patients. In the last case a combined pattern pulmonary fibrosis and emphysema was recorded.

All patients experienced therapies with synthetic or biologic DMARDs before TCZ. Twenty-five patients (89.3\%) have been previously treated with methotrexate (MTX) and 10 with leflunomide (37\%); among biologic DMARDs, 10 patients (37\%) experienced a therapy with TNF inhibitors, 7 with rituximab (25.9\%) and 3 with abatacept (10.7\%).

Twenty-three patients taken TCZ as monotherapy, while in 5 patients TCZ was associated to MTX; on the other hand, 20 patients were treated with a low dose of prednisone (5 mg daily or equivalent). All patients but 3 were treated with subcutaneous TCZ, and 6 patients were switched from intravenous to subcutaneous route of administration.

The evolution of lung function and radiology are summarized in Figure 1. 
PFTs were available at baseline and at the end of the follow-up in 25 patients. After a median followup of 30 months, FVC remained stable in 14 patients (56\%), improved in 5 (20\%) and worsened in 6 (24\%). Mean FVC was stable during follow-up (99\%, IQR 27 at baseline and 96\%, IQR 26 at the end of follow-up).

DLCO showed a similar trend, remaining stable in 14 patients (56\%), improving in 5 (20\%) and worsening in 6 (24\%), even though in 3 patients DLCO and FVC showed an opposite trend. Also mean DLCO remained stable during follow-up (58.5\%, IQR 23 at baseline and 57\%, IQR 31.5 at the end of follow-up).

HRCT was performed at the end of the follow-up in all 28 patients, it was stable in 25 cases, worsened in 2 and improved in the latter. The worsening was recorded in patients with UIP pattern, while the only case of improvement was observed in a patient with NSIP pattern.

No differences were recorded according to the duration of follow-up neither to the previous therapies. During the follow-up period, TCZ was withdrawn in 6/28 patients: for inefficacy in 3 cases and for adverse events in the other 3 (no adverse events were correlated to the treatment). No withdrawals were recorded for a worsening of ILD or for infections.

\section{Discussion}

In our study we evaluated the efficacy and the safety of TCZ in a retrospective cohort of patients affected by RA-ILD, showing a good safety and efficacy. The treatment of these patients is challenging, due to the possible role of DMARDs in the progression of the disease and in the development of acute exacerbation (AE). In fact, both MTX and leflunomide have been associated to

ILD progression and development; recently, Conway demonstrated a mild increased risk of respiratory adverse events (but not ILD) in RA patients treated with MTX compared with other conventional and biologic DMARDs ${ }^{21}$.

Moreover, some Authors described a possible class effect of all anti-tumour necrosis factor inhibitors (TNFi) in the new onset or exacerbation of ILD secondary to RA ${ }^{22-29}$. Perez-Alvarez and colleagues 
[27] and the British Society of Rheumatology has specifically cautioned prescribing TNFi to patients with RA-ILD for the supposed increased risk of exacerbation of the ILD ${ }^{30}$.

Nakashita didn't observe an increase of the prevalence of ILD progression in patients with TCZ and abatacept, whereas a prevalence of $3 \%$ of new ILD appearance and $24 \%$ of ILD worsening were described in TNFi users ${ }^{24,29,31}$. On the other side, Curtis et al. found no significant differences in the risk of ILD incidence and its related complications between patients exposed to tocilizumab, rituximab, or abatacept compared with TNFi therapies ${ }^{32}$. Moreover, some case reports have been reported about acute worsening of pre-existing RA-ILD in patients treated with $\mathrm{TCZ}^{33,34}$.

Despite the lack of evidences, the use of MTX is poorly indicated in patients with RA-ILD ${ }^{35-37}$. In this regard, TCZ could represent a possible safe drug in these patients, considering its efficacy in RA also as monotherapy ${ }^{38}$.

In our population the majority of the patients showed a stability of pulmonary function and about $20 \%$ showed an improvement of PFTs and less than a quarter of patients showed a deterioration of lung function. At the same time, only 2/28 patients showed a worsening of HRCT.

On the whole TCZ demonstrated a good safety profile in patients with RA-ILD and a good efficacy on the stabilization of the lung involvement $1^{4,15}$. In small case series, other Authors observed similar eniicacy in RA-ILD patients treated with other biologic DMARDs. In particular, Md Yusof showed the improvement or the stability of ILD in 30/44 RA patients treated with rituximab, however describing a high number of infectious adverse events ${ }^{39}$; more recently, Fernández-Díaz et al described a high rate of improvement of ILD in 63 RA patients treated with abatacept with or without conventional DMARDS ${ }^{40}$. Data on abatacept were confirmed in 55 Japanese RA-ILD patients, despite the Authors observed a deterioration of lung function in patients treated with a combination therapy with $\mathrm{MTX}^{41}$. Finally, no reports have been reported until now about possible involvement in ILD appearance or deterioration for the Janus Kinases inhibitors ${ }^{42,43}$.

We cannot exclude that some biologic DMARDs, such as TCZ, abatacept, RTX, poorly influence the natural clinical history of ILD and our results, in line with recent literature data, could reflect the 
natural evolution of lung involvement in RA. It should be essential, to minimize the risk of progression and acute exacerbation of ILD, reduce the risk of infection, providing the available vaccinations to all patients.

The management of RA-ILD patients remains a critical unmet medical need. Waiting for prospective controlled studies, in patients with RA-ILD should be preferred biologic DMARDs, namely IL-6 inhibitors, abatacept, and probably Janus kinases inhibitors, that have demonstrated a good safety profile in this specific population ${ }^{3,11,15,42,43}$.

\section{Conclusions}

In conclusion, despite the low number of patients investigated, TCZ should be considered a possible therapeutic option in patients with RA-ILD. Despite increasing data tend to exclude a role for MTX in development and progression of $\mathrm{ILD}^{44}$, the possibility to use TCZ in monotherapy without a significant loss of efficacy ${ }^{35}$ allows us to use this drug also in cases with ILD and high articular disease activity where the use of MTX is poorly recommended.

Finally, an early diagnosis of ILD in RA patients is mandatory to understand the natural history of ILD, its possible predictive factors, and to evaluate the real involvement of some DMARDs, such as ${ }_{v_{\mathbf{I}}} \Gamma \mathrm{X}$, in the development and progression of this severe extra-articular complication ${ }^{45}$. 


\section{References}

[1] Smolen JS, Aletaha D, McInnes IB. Rheumatoid arthritis. Lancet 2016;388:2023-38.

[2] O'Dwyer DN, Armstrong ME, Cooke G, Dodd JD, Veale DJ, Donnelly SC. Rheumatoid Arthritis (RA) associated interstitial lung disease (ILD) Eur J Intern Med. 2013;24:597-603.

[3] Kelly C, Saravanan V, Nisar M, Arthanari S, Woodhead FA, Price-Forbes AN, et al.; British Rheumatoid Interstitial Lung (BRILL) Network. Rheumatoid arthritis-related interstitial lung disease: associations, prognostic factors and physiological and radiological characteristics, a large multicentre UK study. Rheumatology (Oxford) 2014;53:1676-1682.

[4] Bongartz T, Nannini C, Medina-Velasquez YF, Achenbach SJ, Crowson CS, Ryu JH, et al. Incidence and mortality of interstitial lung disease in rheumatoid arthritis: a populationbased study. Arthritis Rheum 2010;62:1583-1591.

[5] Olson AL, Swigris JJ, Sprunger DB, Fischer A, Fernandez-Perez ER, Solomon J, et al. Rheumatoid arthritis-interstitial lung disease-associated mortality. Am J Respir Crit Care Med. 2011;183:372-8.

[6] Hamblin MJ, Horton MR. Rheumatoid Arthritis-Associated Interstitial Lung Disease: Diagnostic Dilemma. Pulmonary Medicine 2011:872120.

[7] Johnson C. Recent advances in the pathogenesis, prediction, and management of rheumatoid arthritis-associated interstitial lung disease. Curr Opin Rheumatol. 2017;29:254-259.

[8] Chatzidionisyou A, Catrina AI. The lung in rheumatoid arthritis, cause or consequence? Curr Opin Rheumatol. 2016;28:76-82.

[9] Roubille C, Haraoui B. Interstitial lung diseases induced or exacerbated by DMARDS and biologic agents in rheumatoid arthritis: A systematic literature review. Semin Arthritis Rheum. 2014;43:613-26.

[10] Jani M, Hirani N, Matteson EL, Dixon WG. The safety of biologic therapies in RA associated interstitial lung disease. Nat Rev Rheumatol. 2014;10:284-94. 
[11] Iqbal K, Kelly C. Treatment of rheumatoid arthritis associated interstitial lung disease: a perspective review. Ther Adv Musculoskel Dis 2015;7:247-267.

[12] Paulin F, Babini A, Mamani M, Mercado J, Caro F. Practical Approach to the Evaluation and Management of Rheumatoid Arthritis-Interstitial Lung Disease Based on its Proven and Hypothetical Mechanisms. Rev Invest Clin. 2017;69:235-242.

[13] Singh JA, Saag KG, Bridges SL Jr, Akl EA, Bannuru RR, Sullivan MC, et al. 2015 American College of Rheumatology Guideline for the Treatment of Rheumatoid Arthritis. Arthritis Care Res. 2016;68:1-26.

[14] Manfredi A, Sebastiani M, Cassone G, Colaci M, Sandri G, Ferri C. Tocilizumab for the treatment of patients with rheumatoid arthritis and interstitial lung diseases: a case series. Clin Exp Rheumatol. 2018;36:342.

[15] Picchianti Diamanti A, Markovic M, Argento G, Giovagnoli S, Ricci A, Laganà B, et al. Therapeutic management of patients with rheumatoid arthritis and associated interstitial lung disease: case report and literature review. Ther Adv Respir Dis. 2017;11:64-72.

[16] Arnett FC, Edworthy SM, Bloch DA, McShane DJ, Fries JF, Cooper NS, et al. The American Rheumatism Association 1987 revised criteria for the classification of rheumatoid arthritis. Arthritis Rheum 1988; 31:315-24.

[17] Aletaha D, Neogi T, Silman AJ, Funovits J, Felson DT, Bingham CO 3rd, et al. 2010 rheumatoid arthritis classification criteria: an American College of Rheumatology/European League Against Rheumatism collaborative initiative. Arthritis Rheum 2010; 62:2569-81.

[18] Travis WD, Costabel U, Hansell DM, King TE Jr, Lynch DA, Nicholson AG, et al.; ATS/ERS Committee on Idiopathic Interstitial Pneumonias; ATS/ERS Committee on Idiopathic Interstitial Pneumonias. An official American Thoracic Society/European Respiratory Society statement: Update of the international multidisciplinary classification of the idiopathic interstitial pneumonias. Am J Respir Crit Care Med. 2013; 188:733-48. 
[19] Karimi-Shah BA, Chowdhury BA. Forced vital capacity in idiopathic pulmonary fibrosis — FDA review of pirfenidone and nintedanib. N Engl J Med 2015;372:1189-91.

[20] Altman DG. Practical Statistics for Medical Research. 1st edn. London: Chapman and Hall, 1991.

[21] Conway R, Low C, Coughlan R, O'Donnell MJ, Carey JJ. Methotrexate and lung disease in rheumatoid arthritis: a meta-analysis of randomized controlled trials. Arthritis Rheumatol 2014; 66:803-812.

[22] Dixon WG, Hyrich KL, Watson KD, Lunt M; BSRBR Control Centre Consortium, Symmons DP; British Society for Rheumatology Biologics Register. Influence of anti-TNF therapy on mortality in patients with rheumatoid arthritis associated interstitial lung disease: results from the British Society for Rheumatology Biologics Register. Ann Rheum Dis 2010; 69:1086-1091.

[23] Hadjinicolaou A, Nisar M, Bhagat S, Parfrey H, Chilvers ER, Ostör AJ. Non-infectious pulmonary complications of newer biological agents for rheumatic diseases-a systematic literature review. Rheumatology (Oxford) 2011; 50:2297-2305.

[24] Koike T, Harigai M, Ishiguro N, Inokuma S, Takei S, Takeuchi T, et al. Safety and effectiveness of adalimumab in Japanese rheumatoid arthritis patients: postmarketing surveillance report of the first 3,000 patients. Mod Rheumatol. 2012;22:498-508.

[25] Panopoulos S, Sfikakis P. Biological treatments and connective tissue disease associated interstitial lung disease. Curr Opin Pulmon Med 2011;17:362-367.

[26] Pearce F, Johnson S, Courtney P. Interstitial lung disease following certolizumab pegol. Rheumatology (Oxford) 2012;51:578-580.

[27] Perez-Alvarez R, Perez-de-Lis M, Diaz-Lagares C, Pego-Reigosa JM, Retamozo S, Bove A, et al. Interstitial lung disease induced or exacerbated by TNF-targeted therapies: analysis of 122 cases. Semin Arthritis Rheum. 2011;41:256-64. 
[28] Schuller A, Coudurier M, Lega JC, Khouatra C, Cottin V, Cordier JF. Interstitial lung disease and anti-TNF-alpha therapy in rheumatoid arthritis: Two different patterns? Rev Mal Respir. 2010;27:232-7.

[29] Takeuchi T, Tatsuki Y, Nogami Y, Ishiguro N, Tanaka Y, Yamanaka H, et al. Postmarketing surveillance of the safety profile of infliximab in 5000 Japanese patients with rheumatoid arthritis. Ann Rheum Dis 2008; 67:189-194.

[30] Ledingham J, Deighton C, British Society for Rheumatology Standards, Guidelines and Audit Working Group. Update on the British Society for Rheumatology guidelines for prescribing TNFalpha blockers in adults with rheumatoid arthritis (update of previous guidelines of April 2001). Rheumatology (Oxford) 2005;44:157-63.

[31] Nakashita T, Ando K, Kaneko N, Takahashi K, Motojima S. Potential risk of TNF inhibitors on the progression of interstitial lung disease in patients with rheumatoid arthritis. BMJ Open. 2014;4:e005615.

[32] Curtis JR, Sarsour K, Napalkov P, Costa LA, Schulman KL. Incidence and complications of interstitial lung disease in users of tocilizumab, rituximab, abatacept and anti-tumor necrosis factor $\alpha$ agents, a retrospective cohort study. Arthritis Res Ther. 2015;17:319.

[33] Kawashiri SY, Kawakami A, Sakamoto N, Ishimatsu Y, Eguchi K. A fatal case of acute exacerbation of interstitial lung disease in a patient with rheumatoid arthritis during treatment with tocilizumab. Rheumatol Int. 2012;32:4023-6.

[34] Wendling D, Vidon C, Godfrin-Valnet M, Rival G, Guillot X, Prati C. Exacerbation of combined pulmonary fibrosis and emphysema syndrome during tocilizumab therapy for rheumatoid arthritis. Joint Bone Spine. 2013;80:670-1.

[35] Saag KG, Teng GG, Patkar NM, Anuntiyo J, Finney C, Curtis JR, et al.; American College of Rheumatology. American College of Rheumatology 2008 recommendations for the use of nonbiologic and biologic disease-modifying antirheumatic drugs in rheumatoid arthritis. Arthritis Rheum. 2008;59:762-84. 
[36] Saravanan V, Kelly C. Reducing the risk of methotrexate pneumonitis in rheumatoid arthritis. Rheumatology (Oxford) 2004;43:143-7.

[37] Rojas-Serrano J, Herrera-Bringas D, Pérez-Román DI, Pérez-Dorame R, Mateos-Toledo H, Mejía M. Rheumatoid arthritis-related interstitial lung disease (RA-ILD): methotrexate and the severity of lung disease are associated to prognosis. Clin Rheumatol. 2017;36:14931500.

[38] Jones G, Wallace T, McIntosh MJ, Brockwell L, Gómez-Reino JJ, Sebba A. Five-year Efficacy and Safety of Tocilizumab Monotherapy in Patients with Rheumatoid Arthritis Who Were Methotrexate- and Biologic-naive or Free of Methotrexate for 6 Months: the AMBITION Study. J Rheumatol. 2017;44:142-6.

[39] Md Yusof MY, Kabia A, Darby M, Lettieri G, Beirne P, Vital EM, et al. Effect of rituximab on the progression of rheumatoid arthritis-related interstitial lung disease: 10 years' experience at a single centre. Rheumatology (Oxford). 2017;56:1348-1357.

[40] Fernández-Díaz C, Loricera J, Castañeda S, López-Mejías R, Ojeda-García C, Olivé A, et al. Abatacept in patients with rheumatoid arthritis and interstitial lung disease: A national multicenter study of 63 patients. Semin Arthritis Rheum. 2018;48:22-27.

[41] Mochizuki T, Ikari K, Yano K, Sato M, Okazaki K. Long-term deterioration of interstitial lung disease in patients with rheumatoid arthritis treated with abatacept. Mod Rheumatol. 2018:1-5.

[42] Cohen S, Curtis JR, DeMasi R, Chen Y, Fan H, Soonasra A, et al. Worldwide, 3-Year, PostMarketing Surveillance Experience with Tofacitinib in Rheumatoid Arthritis. Rheumatol Ther. 2018;5:283-291.

[43] Fleischmann R, Wollenhaupt J, Takiya L, Maniccia A, Kwok K, Wang L, et al. Safety and maintenance of response for tofacitinib monotherapy and combination therapy in rheumatoid arthritis: an analysis of pooled data from open-label long-term extension studies. RMD Open. 2017;3:e000491. 
[44] Manfredi A, Sebastiani M, Cassone G, Fedele AL, Venerito V, Trevisani M, et al. New perspectives in diagnosis of interstitial lung disease related to rheumatoid arthritis. validation study of an electronic stethoscope and ad hoc software for detection of pulmonary crackles. Ann Rheum Dis. 2017;76:248.

[45] Kiely P, Busby AD, Nikiphorou E, Sullivan K, Walsh DA, Creamer P, et al. Is incident rheumatoid arthritis interstitial lung disease associated with methotrexate treatment? Results from a multivariate analysis in the ERAS and ERAN inception cohorts. BMJ Open. 2019; 9:e028466 
Figure 1. Evolution of lung function and radiology during follow-up.

This article is protected by copyright. All rights reserved. 
Median age

Female/Male ratio

Disease duration

ILD duration before TCZ therapy (months)

Follow-up (months)

Rheumatoid factor

ACPA

HRCT pattern

$\begin{array}{lc}\text { UIP } & 14(50 \%) \\ \text { NSIP } & 13(46.5 \%) \\ \text { CPFE } & 1(3.5 \%)\end{array}$

Forced vital capacity (\%)

Diffusion lung CO (\%)

Use of cDMARDs before TCZ

$28(100 \%)$

Mehotrexate

$25(89.3 \%)$

Leflunomide

$10(37 \%)$

TNFalpha inhibitors

$10(37 \%)$

Rituximab

7 (25.9\%)

Abatacept

$2(7.4 \%)$

mCZ mono-therapy

$\mathrm{TCZ}+$ methotrexate

Corticosteroids $20(71.4 \%)$

Continuous data are reported as median (IQR).

ACPA: anti-cyclic citrullinated peptides antibodies, UIP: usual interstitial pneumonia, NSIP: nonspecific interstitial pneumonia, CPFE: combined pulmonary fibrosis and emphysema, cDMARDs: conventional diseases modifying antirheumatic drugs, TCZ: tocilizumab, IQR: interquartile range. 
Evolution of lung function and radiology
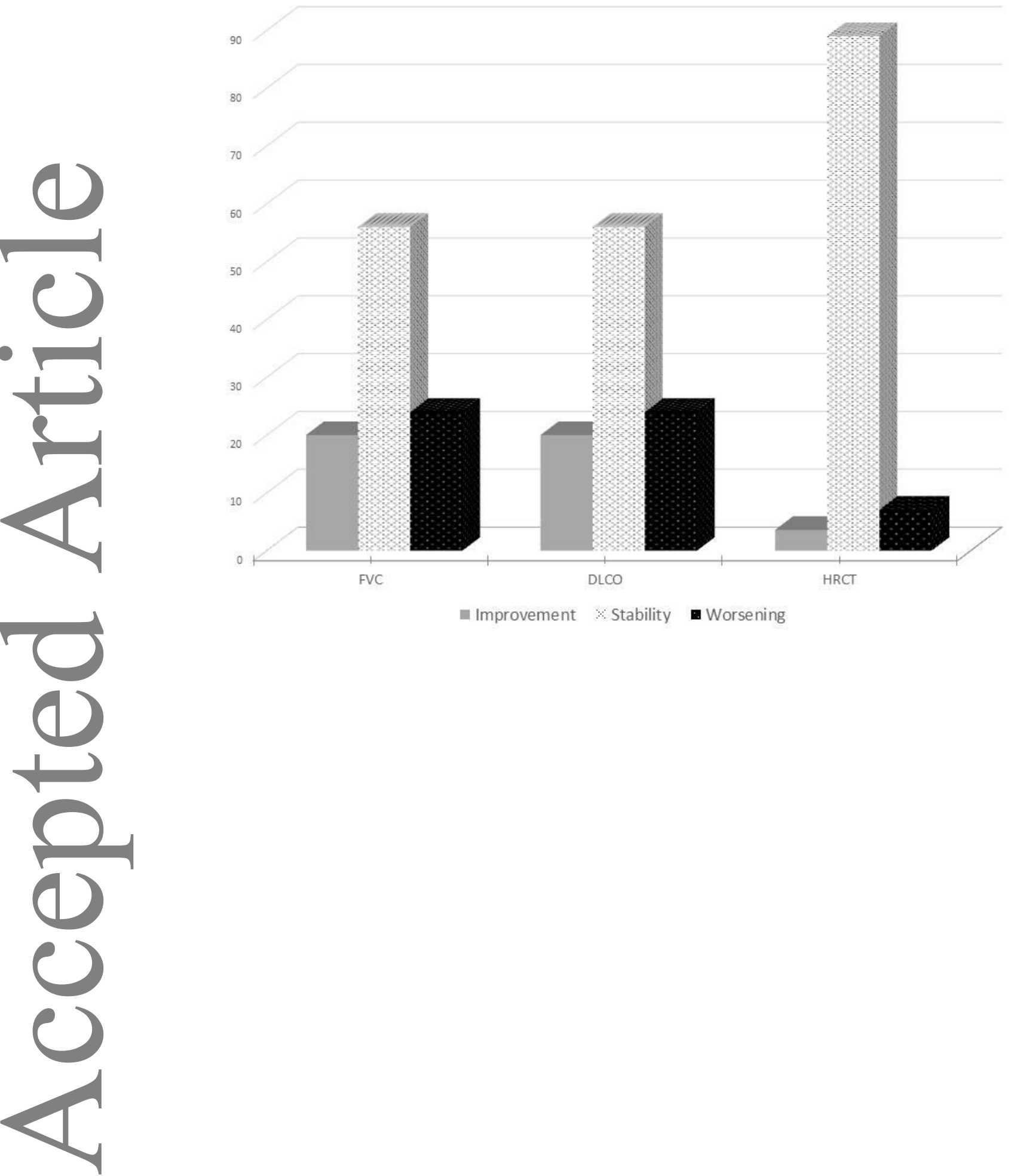